This item was submitted to Loughborough's Research Repository by the author.

Items in Figshare are protected by copyright, with all rights reserved, unless otherwise indicated.

\title{
Concussion, chronic traumatic encephalopathy, and the medicalization of sport
}

\section{PLEASE CITE THE PUBLISHED VERSION}

https://doi.org/10.4324/9780429028175

\section{PUBLISHER}

Routledge

VERSION

AM (Accepted Manuscript)

\section{PUBLISHER STATEMENT}

This is an Accepted Manuscript of a book chapter published by Routledge in Sociocultural Examinations of Sports Concussions on 2019-11-18, available online: http://www.routledge.com/9780367134501

\section{LICENCE}

CC BY-NC-ND 4.0

\section{REPOSITORY RECORD}

Malcolm, Dominic. 2019. "Concussion, Chronic Traumatic Encephalopathy, and the Medicalization of Sport". figshare. https://hdl.handle.net/2134/11871495.v1. 


\section{Concussion, Chronic Traumatic Encephalopathy, and the Medicalisation of Sport}

\section{Dominic Malcolm}

This chapter explores the development of concussion and CTE as issues in sport, through the lens of medicalisation. It argues that medicalisation processes are evident at three distinct levels: namely at a conceptual, institutional and interactional level. Consequently we see: 1 ) that biomedical vocabularies have come to structure public understanding of concussion through, e.g. discussions of the power of neuroscience to identify CTE; 2) that biomedical practices are used to "manage" concussion through, e.g., the institutionalisation of diagnostic and return to play protocols; and 3) that biomedical actors have become centrally placed as the ultimate "cure" of the social problem of concussion through, e.g., and assertion of the primacy of "individual clinical judgement" in interpreting and applying concussion guidelines. The paper explores the dynamics and consequences of these processes, highlighting how the unevenness of these process with medicalisation at the conceptual level being considerably more extensive that the efficacy of treatment at the interactional level would warrant. Moreover, it suggests that the disparity between in the extent of moderation over different domains is partly responsible for the heightened level of social concern in relation to concussion and CTE. The chapter concludes with some practical reflections on the consequences of this medicalisation process, locating the enabling and constraining effects within a framework of medical ethics. 
Sports participants have experienced head injuries of various types since sports participation began. Throughout the early history of sport we find numerous references like the description of the "Brompton gents" who "retired home with broken heads and black eyes" following a cricket match in England in 1731 (cited in Malcolm 2013). But due to the nature of the then contemporary medical understanding, such concerns were largely expressed in relation to the release of blood from the head. Other signs of brain injury - like amnesia, disorientation, or loss of consciousness - because they were/are usually transitory and resolve spontaneously, were not believed to be particularly significant. And it is as a consequence of later modes of medical understanding that firstly concussion, and latterly the potential link with Chronic Traumatic Encephalopathy (CTE), have risen to the fore. It is in light of the temporal specificity of the medical understanding of concussion that any sociocultural examination of this phenomenon must be grounded. This is the fundamental aim of this chapter.

Some excellent work in this regard has already been started (Bachynski 2019; Shurley and Todd 2012). Emily Harrison's (2014) analysis of "the first concussion crisis" shows how doctors treating American college footballers around the turn of the twentieth century began to chart both the frequency of concussion and the potential for long-term behavioural consequences. Harvard team doctors recorded an incidence of almost one concussion per game during the 1906 season. The impact of such concerns was inhibited by the (continued) absence of visual evidence of damage and the empirical evidence of a link between concussion and the subsequent "insanity" and "alcoholism" that some claimed it entailed. However, ultimately the crisis was averted through the mobilisation of economic interests, as colleges began to see sport as an important recruitment tool and attempts were made to emphasise the capacity of football to develop important masculine qualities. Some evidence of the scale of harm was hidden from public view, while technical "solutions" such as enhanced protective equipment and improved playing technique meant that the weaknesses of the body of biomedical evidence could be exploited and the issue dismissed.

Others have rightly pointed to the more sustained and to some extent successful campaigns against boxing. The health hazards of boxing first became subject to explicit and formalised medical concern in 1893. In this year, The Lancet (a leading UK medical journal), drew 
readers' attention to the death of three boxers, all of whom had experienced head injuries of some form (Sheard 1998). The key biomedical development, however, occurred in 1928 when Harrison Martland introduced the term "punch drunk" to describe former boxers who exhibited symptoms of neurological deficit. Martland could only speculate about the underlying damage, but the medical community's adoption of the term dementia pugilistica in 1937 shows that the ideas nevertheless gained widespread acceptance (Castellani and Perry 2017). Indeed, Weinberg and Arond (1952), in one of the founding studies in the sociocultural analysis of sport, described the prevalence of long term neurological damage as a salient feature of the "occupational subculture of boxing." In the UK, the 1969 "Roberts Report" not only concluded that there were approximately 200 severely "punch drunk" boxers in the country, but correlated incidence with career length and fight exposure. Given how long and widely these ideas have been accepted, and their degree of "seriousness," it is remarkable how little attention sociologists have paid to the lived experience of brain injury amongst current and former sports participants.

In what follows I present a developmental (as opposed to historical) account of the changing place and role of concussion in sport. This approach is premised on the belief that the distinction between history and sociology is asocial and erroneous (Malcolm 2015) and that both disciplines (if it is indeed correct to separate them), should be fundamentally oriented towards the study of social processes. The framework I deploy to structure our understanding of concussion, and the central process interrogated, is that of medicalisation. First, we explore the concept of medicalisation, before outlining how it has previously been applied to the study of sport. Subsequently we look at the way in which different dimensions of medicalisation are evident in the development of concussion and CTE as major public issues in sport. Specifically, it is argued that the multi-layered processes of medicalisation are often uneven and sometimes competing and contradictory. Moreover, the generalized and somewhat intangible conceptual concerns regarding CTE have created a demand for action that is specific and empirically measurable and thus must be delivered through the policing of concussion at the institutional and interactional levels. This mismatch or incongruence between these different domains of medicalisation serves to fuel social concerns about head injuries in sport. The conclusion reflects upon the consequences 
of the medicalisation of concussion and CTE, particularly in relation to medical ethical principles.

\section{Medicalisation}

Medicalisation can be defined as the process whereby, "more and more of everyday life has come under medical domain, influence and supervision" (Zola 1983, 295). Initially a discourse about medicalisation arose in relation to the growing role of medicine in defining and treating certain 'deviant' practices. Deviance, sociologically speaking, is behaviour that violates social norms and it was on this basis, for instance, that Talcott Parsons conceptualized illness as a form of deviance; that is to say, when someone is socially recognised as being ill, expectations about 'normal' behaviour are suspended (e.g. the ill are excused from working). Latterly, however, medicalisation has been evoked to describe how a range of natural aspects of the life-course (e.g. childbirth and ageing) have moved from the lay sphere of expertise into the domain of medical practice. Thus, sports medicine as a discrete area of practice, could be described as one manifestation of medicalisation; historically contextualised, we see medical specialists replace the athletes and trainers/coaches who have previously been responsible for the treatment of conditions incurred through, or limiting participation in, sport (Waddington 1996). The central role that clinicians are now required to play in making decisions about the "fitness" of those incurring head injuries to play on is an example of the "policing" of deviance that medicine is called upon to exercise in the realm of sport. Injured players are not expected to continue playing, and those with head injuries are positively restricted in their attempts to do so.

Medicalisation "cannot and should not be read as questioning the value of medicine" (Williams et al. 2018, 775) but it can and should focus on both the process and consequences of a phenomenon becoming defined as a medical problem (Conrad 2015). Thus, fundamental questions might include the consequences of using medicine as a tool of social control or medical professional expansion. Illich (1975), for instance, argued that medicalisation meant that the profession not only cured illness and relieved suffering but created iatrogenesis; that is, medically caused (or exacerbated) illness and/or social problems. Sheard's (2003) argument that the introduction of head guards in boxing may 
have successfully reduced cuts to the head but unintentionally increased the incidence of brain injury is a pertinent example of iatrogenesis. But the broader depiction of medicalisation as intentional and imperialistic floundered as it became clear that the ability and ambition of medicine to achieve such goals were easily exaggerated. For instance, De Swaan (1989) introduced the term "reluctant imperialism" to capture the recognition within the medical profession that being called on to adjudicate on social conflicts (e.g. fitness to play sport) created sometimes unnecessary or somewhat "peripheral" problems for the profession and threatened to undermine the sense of collectivism which has historically contributed to medicine's social power. Indicatively, Malcolm (2009) cites clinicians who seriously damaged previously good relationships with key players through the diagnosis of concussion, thereby weakening their status and occupational security within what is already a notably precarious position (Malcolm 2006).

It has more recently been suggested that while the broader medicalisation process continues to develop (if not extends in terms of reach and speed with which "issues" are medicalised), emphasis has shifted as the "engines of medicalization" proliferate and doctors move from drivers to "gatekeepers" of the process (Conrad 2007, 142). Consequently, medicalisation has come to be understood as potentially evident on three different levels: conceptual, institutional and interactional (Conrad 1992). Respectively, these are evident when: biomedical vocabularies come to structure public understanding of particular aspects of social life; biomedical practices are used to administer social problems; and biomedical actors become central to the "cure" of social problems (Halfmann 2011). In any analysis of these phenomena, we should also be cognisant of the relative willingness of groups to embrace medicalisation and their abilities to resist. Considered in this multidimensional way, medicalisation can be: partial or extensive; the intended or unintended outcome of human action; and/or occur either with or without the medical profession. Different branches of medicine are differentially equipped to achieve medicalisation, and potentially differentially oriented in their aspirations to seek medicalisation.

It is important, therefore, to see medicalisation as a process that has both enabling and constraining effects. The absence of medicalisation - where medical recognition is withheld, research funding is limited, treatments are underdeveloped, and public sympathies are 
muted - can be equally if not more incapacitating and distressing for those involved. The frustrations experienced by Mike Webster (the former National Football League player and the first person diagnosed with CTE) as he and his family sought compensation for his declining cognitive state, are a case in point (Fainaru-Wada and Fainaru 2013). Similarly, it is important to avoid the representation of medicine as a monolith. The sub-speciality of sports medicine holds an ambiguous position in relation to the broader profession. Not only has its status been weakened by its historic commitment to multidisciplinarity and limited by only somewhat partial state support in many Western nations (Malcolm 2017), but it is also necessarily linked to the division of medicine into specialisms and thus subject to intraprofessional as well as interprofessional conflicts (Malcolm and Scott 2011). Specifically in relation to concussion and $\mathrm{CTE}$, for instance, we see elements of friction between the sports medicine community and that of neurology and neuroscience (see, e.g., Sharp and Jenkins' (2015) call for the largely sport-related term concussion, to be withdrawn from clinical use and replaced with a single classification system for traumatic brain injury (TBI)). Thirdly, we must be cognisant of social resistance to medicalisation that this critique has invoked, ranging from the proletarianisation of medicine (changing work conditions, de-skilling through an extended division of labour) to de-professionalisation (greater degrees of administrative control, jurisdictional contestation by members of other healthcare professions, as evidenced by the burgeoning market for books providing complementary and alternative medical treatments for concussion). Finally, it is essential to see the degree of interdependence that is fundamental to the medicine-sport relationship. Sports medicine, as both an area of scientific scholarship and a community of practitioners, is highly dependent on the sport community for both its existence and validation. More recent moves towards the public health potential of exercise have served to expand sports medicine's social influence through a broader public health remit, but it remains the case that without the access to special populations that "sport" can grant, and the social status derived from working with an extremely high profile group of celebrities, sports medicine would have a much more limited aura of expertise.

\section{The medicalisation of sport}


The application of medicalisation to sport emerged in Waddington and Murphy's (1992) analysis of the development of the use of performance-enhancing drugs. As one of the first attempts to understand "doping" in relation to the "supply" of medical assistance (rather than simply athlete demand), it drew on a conceptualisation of medicalisation as the expanding realm of medical influence (although equally a case could have been made here for medicalisation in the sense of the requirement of medicine to take a central role in the policing of this "deviant" behaviour). The publication of this text coincided with that of John Hoberman's seminal developmental account of sports medicine, Mortal Engines (1992), and the two theses are highly compatible. Hoberman $(1992,19)$ for instance, notes that "sport and medicine ... developed a symbiotic relationship during the twentieth century," first with athletes being used as extreme or atypical cases that revealed the limits of human physical potential, and latterly with medical experimentation advancing the performance-orientation of elite sport. Waddington's (1996) subsequent analysis of the development of sports medicine literature illustrates how, in line with this process, athletes came to be defined as a distinct population whose need for continuous medical support is akin to the needs of the chronically ill; in other words, the widespread acceptance of the idea that, "athletes require routine medical supervision, not because they necessarily have a clearly defined pathology but ... simply because they are athletes" (Waddington 1996, 197). Latterly Beamish and Ritchie $(2006,8)$ extended the reach of this argument, suggesting that the rational pursuit of enhanced human athletic performance (and the quest for commercially-rewarded and/or nationally prestigious victory), has led sport to become "an intensive, exhaustive occupation where athletes are fully embroiled in sophisticated training regimes utilizing scientifically developed technologies that create long-term physiological and personality changes as they progress through the high-stakes, "winner-takes-all" road to the pinnacle of world-class sport." For Beamish and Ritchie, therefore, medicalisation is not simply a discrete element of the development of sport, but a fundamental driving force of its contemporary development.

Other examples of the medicalisation of sport include the (mandatory) health screening programmes operant in certain (mainly European) nations, the introduction and development of various forms of participant sex-testing, and the systems of disability classification which underpin the very existence of Paralympic sport. Sport, Medicine and 
Health: the medicalization of sport? (Malcolm 2017), provides the most extensive interrogation of the relationship between sport and medicalisation produced to date. Following a broad review of the interdependence of sport, medicine and health, embracing the organisational development of sport (and exercise) medicine, the provision and practice of elite sport medicine, the role of exercise in public health campaigns, the response of target populations to such campaigns, and the specific issues relating to concussion and cardiac screening, the book concludes that:

The sum of these processes is that the medicalization of sport is far more apparent (or at least extensive) at the conceptual level than it is in relation to the actual practice of medical techniques and/or the engagement of patients ... Constraining the more holistic medicalization of sport has been the triad of sport's relative autonomy, intramedical conflicts which have retarded the development and restricted the jurisdictional domain of sports medicine and state concerns to harness escalating healthcare costs. Medicine has, therefore, been more central to defining aspects of sport using its own nomenclature and paradigmatic assumptions than it has been in public intervention and implementation (Malcolm 2017, 168-169).

Public debates regarding, concussion have exponentially grown since this work was published. Significant here is the connection between concussion and CTE which began, as noted above, in relation to boxing and American football, but which now engulf a range of contact and collision sports and all the major football codes: namely association (or soccer), rugby (union and league) and Australian Rules football. What many now perceive as a crisis in sport has spread both in geographical terms but also in terms of both potential causes and consequences. Which particular aspects of these activities - from high force head-on collisions, to the relatively minor (but potentially frequent) impacts incurred when heading a 430-gram soccer ball - lead to which particular consequences - from short lived and temporary forms of neurocognitive disruption to longer term, life changing conditions such as dementia or Amyotrophic Lateral Sclerosis (ALS) - remains to be fully established. For instance, in a recent 'call for balance when reporting CTE', Stewart et al. $(2019,231)$ noted that 'CTE has yet to be fully defined ... [the] diagnostic criteria are no more than preliminary. We have an incomplete understanding of the extent or distribution of pathology'. Fundamentally though, the potential for activities which have historically been assumed to 
be "healthy" to lead to dementia - described as "one of the global health priorities of our age" (Hillman and Latimer 2017, 1) - has only increased the relevance of an analysis based on the concept of medicalisation. Indeed, what makes these conditions stand out as examples or exemplars of this process is the degree to which they resonate with each level of the broader social process of the medicalisation of sport. To illustrate this, we explore how the medicalisation of concussion and CTE is evident at the conceptual, institutional and interactional levels of analysis.

\section{The Medicalisation of Concussion and CTE}

\section{Conceptual level}

The conceptual level of medicalisation is evident when biomedical vocabularies come to structure public understandings of a particular phenomenon. Because modern societies are so heavily influenced by media representations, the latter inevitably play a very significant part in the dissemination of these biomedical ideas to lay audiences. Thus, analyses of the portrayal of some of the higher profile cases of concussion have highlighted the role of the media in disseminating certain messages about concussion. McGannon et al. (2013), for instance, explored the representation of the National Hockey League's (NHL) Sidney Crosby and his return to play following concussion. They argue that a somewhat "limited" understanding of concussion emerged (in relation to the depiction of the condition as an essentially physical injury with physiological rather than psychological effects), but equally that the public were exposed to messages about both the limitations of existing biomedical understanding, and the potential seriousness, of concussion. Combined, these created the necessity to raise awareness among the general public, and the media took this opportunity to open up a wider political debate about whether or not enough was being done to address the issue. In defining Crosby's rehabilitation in terms of neurological monitoring of physiological symptoms, concussion was fundamentally positioned within biomedical terms rather than the psychological, social, and cultural domains that provide a more holistic perspective to what remains a relatively poorly understood condition (McGannon et al. 2013). 
The degree to which biomedical notions come to structure public understanding is, however, more directly evident in the content of social media due to the more democratic authorship of content. A number of studies have, for instance, identified how Twitter has become a significant medium for concussion-related content, with major themes being users' tweets about their own personal experiences of head injury, news about incidence among professional athletes, dissemination of biomedical research and debates about policy and injury prevention (Workewych et al. 2017). The growth in the quantity of concussion-related social media content can be seen by comparing the surveys of Sullivan et al. (2012) who identified 3488 tweets in 7 days in July 2010, with Workewych et al. (2017) who discovered nearly 300,000 tweets within a month (9 June- 9 July) just three years later (thought notably both studies took place in the off-season for the NHL, NFL, and northern hemisphere rugby union which have, perhaps, had the highest profile concussion incidents). While the vast majority of tweets (83\%) use medical rather than colloquial descriptors (Workewych 2017), a number of researchers have expressed concern about the accuracy of the content disseminated (Ahmed et al. 2017; Kuhn et al. 2017; Workewych et al. 2017).

Such concerns are to some extent predicated on a misplaced degree of faith in the certainty of medical knowledge about these conditions. Indeed, the media surveys conducted by biomedically-oriented researchers complain of both the way the media downplay the seriousness of concussion (e.g. the use of modifying terms such as "blow to the head" to project potentially "softer" implications of the injury, Ahmed and Hall 2017) and the "propogat[ion of] an agenda of one-sided headline news and a sensationalised state of fear" (Kuhn et al. 2017, 1732). But reporting inaccuracies and sensationalism are intertwined as evidenced by the somewhat contradictory outcomes of athlete resistance to concussionrelated public health campaigns (see discussion of medicalisation at the interactional level) and the public self-diagnoses of CTE amongst former elite athletes (Ventresca 2018). Via the media, these athletes often perceive post-playing behavioral, cognitive and emotional experiences behavioural as attributable to CTE yet, as Caron et al. (2013) note, there is a considerable degree of overlap between the documented symptoms of post-concussion syndrome, and the experience of retirement (depression, anxiety, isolation, and even suicidal ideation). These instances are indicative of the potential for the iatrogenic effects of medicalisation where limited and uncertain biomedical knowledge creates a framework 
through which what may be relatively routine aspects of the life-course (i.e. retirement) are interpreted as the initial symptoms of not only a longer-term chronic illness, but one of the most feared conditions of our age. isat

Despite the ambiguities and inconsistencies evident across media representations, and the ultimate privileging of scientific understandings within these accounts (Ventresca 2018), are key markers of medicalisation at the conceptual level. The media are particularly keen to focus on technological "solutions" for concussion, such as the development of wearable impact-measuring equipment, virtual reality concussion assessment tools, and the illusive search for a biomarker of concussion (Wilson 2019). What these developments have in common is an underpinning assumption that concussion will, eventually, be knowable and understandable as a specific corporeal condition amenable to biomedical control. They are premised on the belief that the more precise understanding of the effects of external forces on the brain, or a reliable diagnostic tool are potential outcomes. Yet history shows us that biomedicine is a field in which uncertainty is endemic (Fox 2000). Indeed, advances in the biomedical understanding of concussion have been notably slow in recent decades and many of the changes made to concussion consensus statements are more the product of social concerns than shifting empirical evidence (Malcolm 2019; see next section).

In order to comprehend public understandings of concussion and CTE then, it is important to locate media discourses within the "neuroscientific turn in contemporary biomedicine" (Ventresca 2018,4). Developments in neuroscience are perceived as having the potential to unlock the final frontier of medical understanding. It relies on representing the functioning of the brain in particular ways and while CTE is by no means at the forefront of these processes, being both unamenable to modern imaging techniques and only diagnosable post-mortem, the prominence of the Boston University "brain bank" studies in the public imagination is clear evidence of the foregrounding of biomedicine. The caution which even this group of researchers uses when discussing their research findings (specifically in acknowledging the absence of proof relating to a causal link between CTE and any specific activity or outcome, e.g. Mez et al. 2017), is often disregarded in the simplified accounts disseminated to and by the public on social media. Consequently, the key "causal link" question both exaggerates the power of (contemporary) science to explain all aspects of the 
human condition, and allows the defenders of sporting practices to defer implementing radical changes until such time as "proof" is available. Indicative of the power of this conceptual level of medicalisation has been the successful legal action brought forth by NFL players, for although the case was settled in the absence of compelling evidence of a sportconcussion-CTE link, an explicit assumption was that it was merely a matter of time before neuroscience would produce such compelling evidence (Hardes, 2017).

\section{Institutional level}

Institutional level medicalisation is evident where biomedical practices are used to "manage" corporeal conditions. The initial moves towards the medical management of concussion were, however, highly problematic. For instance in the late 1990s it was argued that existing research on concussion in sport was "anecdotal ... bizarre rather than reflecting established medical principles" and that the field was plagued by a "neuromythology" derived from folk wisdom, methodologically flawed medical research, and media exposés of athletes' experiences of head trauma (McCrory 2001, 82). It was further noted how the disjointed development of the field had become counterproductive to effective medical management. Specifically, the design and publication of an extensive range of severity scales for assessing the condition provided contradictory advice about whether concussion injuries should be graded according to the presence/absence of particular symptoms (rather than their duration), and led to confusion among clinicians about which advice to follow when managing the condition (McCrory 1999).

At the beginning of the twenty-first century, however, a process began whereby the biomedical science of concussion would become more unified and thus coherent. The single most tangible marker of this was the establishment in 2001 of a series of international conferences on sports concussion which have produced a succession of Agreement/Consensus Statements on Concussion in Sport. Initially organised under the auspices of the International Ice Hockey Federation, the Federation Internationale do Football Associations (FIFA) and International Olympic Committee, these consensus statements have led to a degree of regulatory convergence across sports. The production of Concussion in Sport Guidelines (CISG) has led to a standardised definition of concussion 
which, significantly, concludes that a direct blow to the head and loss of consciousness (LOC) are not to be deemed diagnostic prerequisites of concussion (indeed it is frequently cited that approximately only $10 \%$ of concussions entail LOC, e.g. Broglio et al. 2010), . The management protocol produced by the CISG is adopted by most international sports governing bodies and is therefore the global "gold standard." It recommends that athletes: undergo baseline cognitive testing; have concussion symptoms assessed using a standardised Sports Concussion Assessment Tools (SCAT, now in its fourth iteration and more detailed and extensive than ever); and be wholly asymptomatic throughout a sixphase graduated RTP programme, lasting a minimum of six days, and overseen by a medical practitioner. A fundamental logic of these protocols, therefore, is that while there is a need to protect individuals from incurring further damage when concussed (i.e. second impact syndrome) there is no need to protect individuals from incurring multiple concussions over an extended period of time (i.e. CTE).

In light of the prior discussion of the evidential basis for CTE (and how this enables resistance to calls for the implementation of radical changes in sport), it is interesting to note that there is relatively little empirical evidence to substantiate the concussion protocols now in place. Criticisms have been voiced, for instance in relation to: a diagnostic inclusion criteria that are too broad and therefore likely to capture multiple other conditions; the lack of supportive evidence for prescribing physical and cognitive rest; and a flawed graduated RTP protocol that is not operational because most people are never fully asymptomatic of the inclusion criteria (Craton and Leslie 2014, 93). Even the authoritative consensus statements have noted that the "science of concussion is evolving" (McCrory et al. 2013,250$)$ and the consensus statements are replete with provisos and reservations stressing how many areas of concussion science contain uncertainties.

But such flaws and evidential weaknesses have not stopped the widespread adoption of these protocols across the sports community, albeit with some sport-specific modifications. For instance, in 2011 the Australian Football League (AFL) and National Rugby League (NRL) implemented concussion management systems based on the CISG. To encourage conservative management, rugby league teams were awarded an additional substitution for when a player is withdrawn exhibiting concussion symptoms. Rugby union has, too, adopted the CISG but supplements this protocol with the more contentious "head injury bin" which 
permits temporary substitutions to create extra time for the diagnosis of concussion. Ironically, given FIFA's early involvement in the process, the adoption of the CISG in football has been relatively slow. For instance, the English Football Association only adopted use of the SCAT and the six-phase graduated RTP (although not time-limited) in its 2012-13 regulations, and it was not until a number of high profile incidents (particularly involving the Tottenham Hotspur goalkeeper Hugo Lloris who completed a match after having clearly been knocked unconscious for a significant period), that the regulations were revised to mirror the "gold standard" protocol noted above. It is testimony to the power and authority of the medical profession, and indicative of how socially pervasive the medicalisation process is, that these protocols, based on little more than the ethical principle of "first do no harm" are so widely adopted.

Perhaps the clearest indication of the medicalisation of concussion at the institutional level, however, is the licence and mandate granted to these biomedical practices through state legislature. At the forefront of this development has been the Lystedt Law which, since its introduction in Washington state in 2009, has been adopted across the US. While there is considerable cross-state diversity in the specific terms of this concussion legislation, implemented measures include training/education of coaches, players and parents, the compulsory notification of parents when a concussion is suspected or diagnosed, and a requirement that players must be cleared by a licensed physician or someone trained in brain injury management before returning to play. Proposals to introduce a parallel Rowan's Law in Canada illustrate this as an on-going process.

\section{Interactional level}

Interactional levels of medicalisation are evident when biomedical actors become centrally placed as the ultimate "cure" of the social problem of concussion. Indicatively, the various SCAT tools are premised on the identification of physicians as the appropriate professionals to take responsibility for concussion management (a role augmented by the increasing complexity of the SCAT). Most fundamentally, perhaps, the primacy of medicine is established by the continued provision in the CISG of all RTP decisions to fundamentally remain a matter of individual clinical judgment (McNamee et al. 2015). But remarkably, this 
jurisdictional domain has been established in relation to a condition which does not seem to require esoteric skills. The symptoms of concussion - memory loss, dizziness, loss of consciousness - are not hard to detect although, ironically, the condition is now claimed to be, "considered among the most complex injuries in sports medicine to diagnose, assess and manage" (McCrory et al. 2013, 7). Arguably, what makes diagnosis problematic is not the specialist medical knowledge required to recognise the signs and symptoms of concussion, but the pressures experienced in the context of elite level sport where players and coaches are highly resistant to being withdrawn from play (although studies show that schoolchildren exhibit similar non-compliance (e.g. Kroshus et al. 2015; Mrazik et al. 2015)). What we see, therefore, is the development of an essentially lay-world problem of policing injury/deviance incorporated into the medical armoury, as diagnosis leads to enforced withdrawal from sport, the imposition of physical and cognitive rest, and thus the suspension of expectations of "normal" behavior. Claims about complexity serve to justify more extensive medicalisation.

Regulatory changes have progressively reinforced this process. While the initial CISG recommendation for the withdrawal of players exhibiting any symptoms became superseded by the less conservative emphasis on medical intervention when cases of concussion have explicitly been diagnosed, importantly the former was essentially amenable to lay assessment while the later places concussion squarely within the domain of medical jurisdiction. The implementation of such guidelines further shifts the balance of jurisdictional duties between first aiders/sports trainers and physicians. Partridge (2014) notes that AFL and NRL regulations require players suspected of sustaining a concussion to be assessed by a first-aider yet also that players "need an urgent medical assessment by a medical practitioner" because "the management of head injury is difficult for non-medical personnel" (Partridge 2014, 67 \& 68). Moves in various leagues to employ "concussion spotters" in the stands, or deploy an independent concussion expert to support team physicians in their concussion diagnoses, are practices premised on the belief that the complexity of concussion (as evidenced by medical uncertainty) necessitates medical monopoly.

Despite the developments which centrally position biomedical actors in the "solution" to concussion, there is a marked degree of subcultural resistance which means that, of the 
three levels of medicalisation that are potentially evident, it is at the interactional level that the process is weakest. A number of biomedical studies suggested that the reluctance to consult with medical staff (which is not unusual in sport or even in the broader population), is particular marked in relation to athletes and brain injuries, and ascribed various reasons for this behaviour, including athletes': (a) perceptions that their condition is not serious enough, (b) reluctance to leave the game and/or let down teammates, or (c) disbelief that a concussion has occurred (Broglio et al. 2010; Fraas, et al. 2013; McCrea, et al. 2004). But Malcolm's (2009) study of elite rugby explained how attitudes towards concussion were shaped by the individual's perception that their trajectory of recovery was undetermined and could have an unpredictable impact on their lives. Athletes reported that concussions did not necessarily impair playing performance, but uncertainty over what might happen if they revealed their concerns (would they, for instance, regain their place on the team?) meant that athletes were reluctant to withdraw from sporting activities. Uncertainty also informed their decisions about whether to consult medical staff. Players knew that in most cases clinicians could not offer relief from symptoms or enable their recovery and return-toplay. They also knew that alerting clinical staff to their concerns about having suffered a concussion would likely only disrupt their careers and identities through enforced withdrawal from sporting activities.

Most recently, Liston et al. (2018) documented athletes' distinctly risky behaviours in relation to sport-related concussions. This study of Irish amateur rugby players showed that individuals managed concussions by downplaying, ignoring, denying the significance, or concealing symptoms and "playing on". Most significantly, players spoke of their "preference" for experiencing a concussion rather than a musculoskeletal injury, arguing that the former frequently entailed a more limited impact on a person's sporting performance. They even rationalised the experiences of concussion-induced cognitive impairment as "reverting" the individual to a "primal state" and thus facilitating the kind of aggression that (many believed) contributes to rugby sporting performance. Severity of injury was assessed in relation to the length of time one was unable to play sport, and excluded considerations of longer term neurocognitive decline. This "head strong" attitude was derived "from within the subculture of rugby, that is, originating in the level of commitment made by players to each other and to the game" (Liston et al., 2018, 676). 
The consequences of these patterns of athlete behaviour are that clinical autonomy over the management and treatment of concussion tends to be limited. Ultimately, clinicians have to negotiate the implementation of diagnostic and treatment guidelines so that they do not exacerbate patient non-compliance. Consequently, studies suggest that clinicians may seek to individualise concussions (considering how that particular player has responded to head injuries in the past) and so remove diagnosis away from the necessarily generalised regulations that characterise the medicalisation process (for examples, see Malcolm 2009; 2018). Alternatively, clinicians sometimes rationalise the use of their own experience to enable personal "guidelines" to supersede standardised diagnostic protocols. Both strategies effectively enable them to operationalise an understanding of concussion in ways that are most recognised and accepted by their "clients." They may, for instance, seek to avoid an explicit diagnosis of concussion and invoke alternative rationales to remove a player from play. They may, alternatively, prioritise LOC in the diagnostic criteria because their experience has shown them that this is least likely to lead to conflict with players and coaches. Ultimately many clinicians modify their understanding of concussion and their treatment of players because their status and security in these settings cannot be guaranteed (Malcolm 2009). Through such actions the "disunity" and incoherence of the medicalisation process becomes particularly pronounced.

\section{Conclusion}

Contemporary understandings of concussion and CTE can thus be seen to have been shaped according to medicalisation processes. Moreover, this "case study" illustrates how medicalisation is too far reaching and complex a process to be amenable to the control of a single unified group of medically trained individuals, or to a simple "good-bad" assessment of its consequences. Rather, we need to understand medicalisation as a multi-layered process which is, in many ways, uncoordinated and exhibits a combination of intended and unintended outcomes. Thus, while the medicalisation of concussion (at the interactional level) is ultimately restricted due to the relative autonomy of sport as a sphere of social practice, and the relative inability of medical practitioners to deliver the social value that often underpins the expansion of medicine's jurisdictional domain, it is at the same time accelerated by the longer-term and necessarily less tangible conceptual level concerns 
about CTE. This analysis advances previous work (e.g. Malcolm 2017) by suggesting that not only is the medicalisation of concussion unevenly developed across the three respective domains, but that it is this unevenness that shapes our understanding and heightens social concerns. Specifically, the progression of concerns about concussion and particularly CTE that are being driven at the conceptual level, fuel the calls for action at the institutional and interactional level; demands which, in practice, contemporary biomedicine is unable to satisfy. As de Swaan's (1989) notion of "reluctant imperialism" suggests, the contradictions between the promise of medicine (conceptual) and the reality of delivery (institution and interactional) is not unique to this case. Medicine must continually moderate the public expectations it, demands in part, creates through the profession's own claims for status and influence.

But as noted earlier, none of this should be interpreted as questioning the value or even necessity of medicine's presence in this field. Medicalisation (at the conceptual level) has raised awareness of the potential dangers of taking part in sport, provided (at the institutional level) a more consistent set of tools with which to manage brain injuries experienced by athletes, and (at the interactional level) presents the most tangible currently available source of help to the concussed individual. Some of these, we should remember, suffer considerable hardship and life-changing symptoms.

Rather, justification for the scope of existing medical interventions can be found in the degree to which the four main ethical goals of medicine are met. The beneficence principle of medical ethics, and the pursuit of the relief of human suffering that is at the heart of the scientific discipline and profession of medicine, invoke a duty to attend to particular concerns relating to concussion and CTE. Given the potential link between combat, contact and collision sports, and both short-term cognitive damage and longer-term neurocognitive decline, an element of medicalisation is to be welcomed.

Against this must be a concern about the non-maleficence (or first do no harm) principle of medical ethics, for whilst biomedical understanding of concussion and CTE remains largely exploratory there is a danger that in certain cases the curtailment of a sphere of their social lives which they find particularly meaningful and valuable is unnecessary. Indeed it has been argued that the sports injuries in general, (and thus concussions in particular) are experienced as particularly biographically disruptive and therefore subjectively highly 
problematic (Malcolm and Pullen 2018). Far from suggesting that the influence of medicine over the social experience of concussion has been negative, the principle of beneficence would be greatly enhanced by a broader recognition of the harms routinely done through sports participation.

Thirdly, fundamental questions also need to be raised about equality, for one potential outcome of the debates around concussion and CTE is to consign the health "benefits" of taking part in these activities to the most socially privileged. If, for instance, medicalisation leads to the regulation of sports such that participation is only allowed where a specified (and probably costly) healthcare provision is available, then we may see the restriction of a set of activities which are relatively highly socially valued, to an economic elite. Such exclusionary activities would, in all likelihood, become a further resource of cultural capital which dominant social classes use to reinforce the existing social hierarchy.

Finally, and perhaps most ethically challenging of all is the principle of autonomy. The resistance of sports organisations to the implementation of changes to the way sports are played cannot simply be dismissed as "ostrich-like", head in the sand, behaviour as portrayals such as League of Denial suggest (Fainaru-Wada and Fainaru 2013). While some sports organisations have reacted to proposed rule changes with an apparent ignorance of the histories of the activities which they govern (histories which show that continuous development is the normal state of affairs), equally critics must recognise that considerable justification is normally required to limit the practice of cognisant individuals capable of giving informed consent for actions which will not harm anybody but themselves. A significant consequence of medicalisation may be to undermine the autonomy of individuals to take part in activities which may represent considerable risk of harm to themselves, but which they judge to represent a tangible and significant enhancement of their own life conditions. Despite such longstanding and compelling evidence linking boxing and neurocognitive decline, a fairly compelling ethical distinction (boxing is the only sport where concussion - or loss of consciousness - is a deliberate and fundamental part of competition), and substantial medical support for prohibition, it should be remembered that the sport has only ever been banned in a small minority of countries. More radical regulatory change across a wider range of sports cannot be expected until a more extensive, coherent, and compelling medicalization process has occurred. 


\section{References}

Ahmed, O., and E. Hall 2017. "'It was only a Mild Concussion:' Exploring the Description of Sports Concussion in Online News Articles." Physical Therapy in Sport 23: 7-13.

Bachynski, K. (2019) "The duty of their elders" - Doctors, coaches, and the framing of youth football's health risks, 1950s-1960s', Journal of the History of Medicine and Allied Sciences, 0(0): 1-25.

Beamish, R. and I. Ritchie 2006. Fastest, Highest, Strongest: A Critique of High Performance Sport. London: Routledge.

Broglio, S., Vagnozzi, R., Sabin, M., Signoretti, S., Tavazzi, B., and G. Lazzarino 2010.

"Concussion Occurrence and Knowledge in Italian Football (Soccer)." Journal of Sports Science and Medicine 9: 418-430.

Caron, J., Bloom, G., Johnston, K. and Sabiston, C. (2013) 'Effects of multiple concussion on retired National Hockey League players', Journal of Sport \& Exercise Psychology, 35: 168-179.

Castellani, R. and G. Perry 2017. "Dementia Pugilistica Revisited." Journal of Alzheimer's Disease 60(4): 1209-1221.

Conrad, P. 1992. "Medicalization and Social Control." Annual Review of Sociology 18: 20932.

Conrad, P. 2007. The Medicalization of Society: On the Transformation of Human Conditions into Treatable Disorders. London: Johns Hopkins University Press.

Conrad, P. 2015. "Foreword." In Reimagining (Bio)medicalization, Pharmaceuticals and Genetics: Old Critiques and New Engagements, edited by S. Bell and A.E. Figert, vii-ix. London: Routledge.

Craton, N. and O. Leslie 2014. "Time to re-think the Zurich Guidelines? A critique on the Consensus Statement on Concussion in Sport, held in Zurich, November 2012." Clinical Journal of Sports Medicine 24(2): 93-95.

De Swaan, A. 1989. "The Reluctant Imperialism of the Medical Profession," Social Science and Medicine 28(11): 1165-1170.

Fainaru-Wada, M. and Fainaru, S. 2013. League of Denial. New York: Crown Business.

Fox, R.C. 2000. "Medical Uncertainty Revisited." In The Handbook of Social Studies in Health and Medicine edited by G.L. Albrecht, R. Fitzpatrick and S.C. Scrimshaw), 409-425. London: Sage.

Fraas, M., Coughlan, G., Hart, E. and C. McCarthy 2013. "Concussion History and Reporting Rates in elite Irish Rugby Union Players." Physical Therapy in Sport 15: 136-142.

Halfmann, D. 2011. "Recognizing Medicalization and Demedicalization: Discourses, Practices and Identities." Health 16(2): 186-207.

Hardes, J. 2017. "Governing Sporting Brains: Concussion, Neuroscience, and the Biopolitical Regulation of Sport." Sport, Ethics \& Philosophy 11: 281-293.

Harrison, E. 2014. "The First Concussion Crisis: Head Injury and Evidence in Early American Football." American Journal of Public Health 104: 822-833. 
Hillman, A., and J. Latimer 2017. "Cultural Representations of Dementia." Plos Medicine 14(3): e102274.

Hoberman, J. 1992. Mortal Engines: The Science of Performance and the Dehumanization of Sport. New York, Free Press.

Illich, I. 1975. Medical Nemesis. London: Calder \& Boyers.

Kroshus, E., Garnett, B., Hawrilenko, M. and C. Baugh. 2015. "Concussion Under-Reporting and Pressure from Coaches, Teammates, Fans and Parents." Social Science and Medicine, 134: 66-75.

Kuhn, A., Yengo-Kahn, A., Kerr, Z., and S. Zuckerman 2017. "Sports Concussion Research, Chronic Traumatic Encephalopathy and the Media: Repairing the Disconnect." British Journal of Sports Medicine 51: 1732-1733.

Liston, K., McDowell, M., Malcolm, D., Scott, A. and I. Waddington, I. 2018. “On Being 'Head Strong:': The Pain Zone and Concussion in Non-Elite Rugby Union." International Review for the Sociology of Sport, 53(6): 668-684

Malcolm, D. 2006. "Unprofessional Practice? The Power and Status of Sports Physicians." Sociology of Sport Journal 23(4): 376-395.

Malcolm, D. 2009. "Medical Uncertainty and Clinician-Athlete Relations: The Management of Concussion Injuries in Rugby Union." Sociology of Sport Journal 26(2): 191-210.

Malcolm, D. 2013. Globalizing Cricket: Englishness, Empire and Identity. Bloomsbury: London.

Malcolm, D. 2015. "Elias and the Sociology of Sport." In Routledge Handbook of Sociology of Sport, edited by R. Giulianotti, 50-60. London: Routledge.

Malcolm, D. 2017. Sport, Medicine and Health: the medicalization of sport? London: Routledge.

Malcolm, D. 2019. The Concussion Crisis in Sport. London: Routledge.

Malcolm, D. and Pullen, E. 2018. “'Everything I Enjoy doing I just Couldn't do': Sport-related Injury and Biographical Disruption." Health: An Interdisciplinary Journal for the Social Study of Health, Illness and Medicine http://journals.sagepub.com/doi/pdf/10.1177/1363459318800142

Malcolm, D. and A. Scott 2011. "Professional Relations in Sport Healthcare: Workplace Responses to Organisational Change." Social Science and Medicine 72: 513-520.

McCrea, M., Hammeke, T., Olsen, G. Leo, P., and K. Guskiewicz 2004. "Unreported Concussion in High School Football Players: Implications for Prevention." Clinical Journal of Sports Medicine 14: 13-17.

McCrory, P. 1999. "You can Run by you can't Hide: The Role of Concussion Severity Scales in Sport." British Journal of Sports Medicine 33: 297-280.

McCrory, P. 2001. "When to Retire after Concussion?" British Journal of Sports Medicine 35: 81-82.

McCrory, P. Meeuwisse, W. Aubrey, M. et al 2013. "Consensus Statement on Concussion in Sport: the $4^{\text {th }}$ International Conference on Concussion in Sport held in Zurich, November 2012." British Journal of Sports Medicine 47: 250-258. 
McGannon, K., Cunningham, S. and R. Schinke 2013. "Understanding Concussion in SocioCultural Context: A Media Analysis of a National Hockey League Star's Concussion." Psychology of Sport and Exercise 14: 891-899.

McNamee, M., Partridge, B. and L. Anderson 2015. "Concussion in Sport: Conceptual and Ethical Issues." Kinesiology Review 4: 190-202.

Mez, J., Daneshvar, D.H., Kiernan, P.T., et al. 2017. "Clinicopathological Evaluation of Chronic Traumatic Encephalopathy in Players of American Football." Journal of the American Medical Association 318(4): 360-370.

Mrazik, M., Dennison, C., Brooks, B., Yeates, K.O., Babul, S. and D. Naidu. 2015. “A Qualitative Review of Sports Concussion Education: Prime Time for Evidence-Based Knowledge Translation.” British Journal of Sports Medicine, 49: 1548-1553.

Partridge, B. 2014. "Dazed and Confused: Sports Medicine, Conflicts of Interest, and Concussion Management." Bioethical Inquiry 11: 65-74.

Sharp, D. and P. Jenkins 2015. "Concussion is Confusing us All." Neurology 15: 172-186.

Shurley, J. and J. Todd 2012. "Boxing Lessons: An Historical Review of Chronic Head Trauma in Boxing and Football." Kinesiology 1, 170-184.

Sheard, K. 1998. "'Brutal and degrading:' the Medical Profession and Boxing, 1838-1984." International Journal of the History of Sport 15(3): 74-102.

Stewart, W., Allinson, Al-Sarraj, S. et al. 2019. "Primum non Nocere: A Call for Balance when Reporting CTE." Lancet Neurology, 18: 231-232.

Sullivan, S., Schneiders, A., Cheang, C., Kitto, E., Lee, H., Redhead, J.et al. 2012. "'What's happening?' A Content Analysis of Concussion-related Traffic on Twitter." British Journal of Sports Medicine 46, 258-263.

Ventresca, M. in press. "The Curious Case of CTE: Mediating Materialities of Traumatic Brain Injury." Communication and Sport.

Waddington, I. 1996. "The Development of Sports Medicine." Sociology of Sport Journal 13(2): 176-96.

Waddington, I. and P. Murphy 1992. "Drugs, Sport and Ideologies." In Sport and leisure in the civilizing process, edited by E. Dunning and C. Rojek, 36-64. London: Macmillan.

Weinberg, S.K. and H. Arond 1952. The Occupational Culture of the Boxer." American Journal of Sociology 62: 460-469.

Williams, S.J., Coveney, C. and J. Gabe 2018. "The Concept of Medicalisation Reassessed: A Response to Joan Busfield," Sociology of Health and IIIness 39(5): 775-780.

Wilson, J. (2019) "Premier League Players to Assist in Research into Concussion," The Daily Telegraph, January 16, https://www.telegraph.co.uk/football/2019/01/16/premierleague-players-assist-research-concussion/

Workewych, A., Muzzi, M., Jing, R., Zhang, S., Topolovec-Vranic, J., and M. Cusimano 2017. "Twitter and Traumatic Brain Injury: A Content and Sentiment Analysis of Tweets Pertaining to Sport-related Brain Injury." SAGE Open Medicine 5: 1-11.

Zola, I. 1972. "Medicine as an Institution of Social Control." Sociological Review 20(4): 487504. 